\title{
Actitudes de los Estudiantes de Formación Docente del Centro \\ Regional de Educación de Pilar sobre el ejercicio de la profesión
}

\author{
Fulvia Karina Gamarra de Velazco \\ gamarrakarina9@gmail.com \\ Pilar - Paraguay
}

\section{RESUMEN}

El presente trabajo se basa en la investigación del tema denominado "Actitudes de los estudiantes de Formación docente del Centro Regional de Educación de Pilar sobre el ejercicio de la profesión". Este estudio tuvo como objetivo general conocer la orientación vocacional de los estudiantes de Formación Docente del Centro Regional de Educación de Pilar sobre la profesión mediante el análisis exhaustivo de la dimensión cognitiva, afectiva y conductual, sobre los alumnos en el contexto de la institución mencionada. El trabajo se llevó adelante en el Centro Regional de Educación de Pilar (CREP) Mariscal Francisco Solano López, de la ciudad de Pilar- Neembucú, en el año 2019, específicamente en el Nivel de Formación Docente, con alumnos matriculados. El enfoque cuantitativo y cualitativo, fueron la orientación metodológica definida para este trabajo de investigación, de alcance explicativo. Para poder recabar las informaciones necesarias, se hizo uso de las entrevistas semiestructuras y cuestionarios, de cuya aplicación surge la información obtenida, sometida a posteriori a un proceso de codificación, tabulación y análisis estadístico. Mediante las técnicas de investigación aplicadas (cuestionarios y entrevistas), se hace referencia que tanto en la dimensión cognitiva y conductual se refleja resultados favorables, cuanto más detallado sea el conocimiento y la asimilación, más favorable será la actitud hacia la profesión. Mientras que en la dimensión afectiva, se identifica que ésta es la dimensión que requiere mayor atención, ya que en más de un reactivo del cuestionario se ha indicado como indiferente o estar en desacuerdo, en tanto que, se reconoce que el aspecto afectivo es un valor preponderante, que incluye sensaciones y sentimientos expresados hacia la profesión, tiene que ver con la comunicación abierta entre los estudiantes y para con los docentes, promoviendo la convivencia social y profesional, activando una interdependencia emocional en las relaciones humanas para fortalecer la formación profesional.

Palabras claves: Actitudes; Estudiantes; Formación Docente 


\section{Attitudes of Teacher Training Students of the Pilar Regional Education Center about the exercise of the profession}

\section{ABSTRACT}

This work is based on the investigation of the topic called "Attitudes of teacher training students of the Regional Center of Education of Pilar on the exercise of the profession". The general objective of this study was to know the vocational orientation of the Teacher Training students of the Regional Center of Education of Pilar on the profession through the exhaustive analysis of the cognitive, affective and behavioral dimension, on the students in the context of the mentioned institution. The work was carried out at the Pilar Regional Education Center (CREP) Mariscal Francisco Solano López, in the city of PilarNeembucú, in 2019, specifically at the Teacher Training Level, with enrolled students. The quantitative and qualitative approach were the methodological orientation defined for this research work, of explanatory scope. In order to collect the necessary information, semi-structured interviews and questionnaires were used, from whose application the information obtained arises, subsequently subjected to a process of coding, tabulation and statistical analysis. Through the applied research techniques (questionnaires and interviews), it is mentioned that both in the cognitive and behavioral dimension, favorable results are reflected, the more detailed the knowledge and assimilation, the more favorable the attitude towards the profession. While in the affective dimension, it is identified that this is the dimension that requires more attention, since in more than one item of the questionnaire it has been indicated as indifferent or to disagree, while it is recognized that the affective aspect is a Preponderant value, which includes feelings and feelings expressed towards the profession, has to do with open communication between students and with teachers, promoting social and professional coexistence, activating an emotional interdependence in human relationships to strengthen professional training.

Keywords: Attitudes; Students; Teacher training

Artículo recibido: 05 de Abril 2021

Aceptado para publicación: 28 de Mayo 2021

Correspondencia: gamarrakarina9@gmail.com

Conflictos de Interés: Ninguna que declarar 


\section{INTRODUCCIÓN}

Las actitudes son adquiridas, son el resultado de las experiencias y del aprendizaje que el individuo ha obtenido a lo largo de su historia de vida, que contribuye a que denote una tendencia a responder de determinada manera y no de otra; pueden ser flexibles y susceptibles a cambio, especialmente si tienen impacto en el contexto en el que se presenta la conducta.

La actitud responde a una orientación o disposición aprendida hacia un objeto o situación, que proporciona una tendencia a responder de manera favorable o desfavorable hacia el objeto o situación. En el ámbito académico, la formación de la actitud en los alumnos se encuentra supeditada al marco institucional, al modelo del sistema educativo imperante y, ante todo, al enfoque, estimulación y generación del docente. El estudiante, de esta manera, asimila el rol profesional dentro de su contexto competitivo, mediante las funciones auto formativas, confirmativas y relacionales asimiladas, en la carrera, que le son transmitidas.

Las actitudes, entonces, hacia la profesión pueden ser formadas mediante múltiples procesos personales y sociales, en el ámbito de los elementos tácitamente presentes en ellas. En efecto, en el estudio de las actitudes profesionales desarrolladas en los alumnos queda asumir el análisis de las dimensiones cognitivas, afectivas y conductual. Esto permitirá conocer de manera amplia y precisa la actitud de los estudiantes de formación docente del Centro Regional de Educación de Pilar sobre la profesión, propósito de la presente investigación.

Desde la perspectiva planteada, las actitudes son juzgadas como elementos de dimensión múltiple que incluyen un amplio rango de respuestas de cualidad cognitiva, afectiva y comportamental. De esta manera se alcanza a conocer facetas nuevas de la conducta humana en relación a la formación profesional en el ámbito académico.

La formación profesional actual se desarrolla atendiendo los principios pedagógicos fundamentales con fines a su implementación en el marco laboral, utilizando los contenidos, tecnologías, recursos y equipos desde la perspectiva de la visión y misión institucional, con miras a un contexto futuro sumamente competitivo.

El progreso individual y colectivo del estudiante se encuentra estrechamente ligado a los modelos institucionales, con las implicancias que ello conlleva, que involucra igualmente al estamento docente, en la confrontación de problemas y retos científicos y tecnológicos 
reales de la sociedad, y del cual los estudiantes se benefician y aprenden en forma evidente y autogestionada.

A pesar de los esfuerzos institucionales y de las fortalezas del sistema, se observa la falta del desarrollo de actitudes o, dicho de otra manera, la formación de actitudes negativas por parte de los alumnos con respecto a la carrera y por sobre todo a su futuro profesional. Cabe recalcar que el desarrollo de actitudes en los alumnos de Formación Docente juega un rol más que importante en su futuro profesional, lo que resulta evidente en el análisis de sus componentes básicos, en las dimensiones cognitiva, afectiva y comportamental.

Pues, en lo que concierne a lo cognitivo, se resalta las falencias en el desarrollo del reconocimiento de tres tipos de conocimientos: el que tiene, el que puede conseguir y el que puede construir con otros. En lo afectivo interviene la comunicación abierta entre los estudiantes y para con los docentes, promoviendo la convivencia social y profesional, activando una interdependencia emocional en las relaciones humanas para fortalecer la formación profesional. El elemento comportamental comprende la acción caracterizada por el dominio de la información actualizada y pertinente, que permite asimilar contenidos programáticos aplicables en la formación profesional y para establecer una comunicación fluida, constructiva y favorable para la interacción formadora entre estudiantes y profesores.

\section{Planteamiento del problema}

Con base a lo anterior, puede apreciarse, que, a pesar de los lineamientos y esfuerzos institucionales, los estudiantes no se encuentran conscientes de la dimensión real de la profesión que abrazan, de sus implicancias, del ámbito de competencia, del mercado laboral, etc.

La falta de actitud hacia la profesión es una constante en los centros de formación docente, debido fundamentalmente a la falta de perspectiva profesional y laboral, la insatisfacción general demostrada por los docentes en ejercicio de la profesión, la falta de aceptación del desempeño docente a nivel social y la firmeza vocacional expresada por la convicción hacia la carrera.

Indefectiblemente contribuye en las actitudes de los alumnos hacia la profesión la transmisión de inseguridad y la falta de responsabilidad educativa de algunos docentes en el incumplimiento o desentendimiento de la aplicación y desarrollo de objetivos básicos tales como la confianza en sí mismo, apertura, responsabilidad, etc. 
La prevalencia de tal situación, lleva irremediablemente a los alumnos al conformismo de permanecer en la institución, sin importar las calificaciones y el promedio alcanzado, haciendo el esfuerzo mínimo por promocionarse al curso superior inmediato, con pocas ganas de aprender y proyectarse, sin ilusión alguna hacia el futuro profesional, esperando oportunidades fortuitas de acceder a espacios laborales en un ambiente cada vez más reducido.

La idea del trabajo como tal surge a partir de dos perspectivas. La primera de carácter intrínseco del autor, vale decir a iniciativa genuina del autor en la búsqueda de respuestas concretas al problema planteado, a la falta de actitud de los alumnos sobre su futura profesión. El segundo aspecto, extrínseco, se refiere al cumplimiento del prerrequisito del curso de post grado, orientado a establecer una investigación referente al área de conocimiento como trabajo de post grado.

El progreso de estudios encarados en el ámbito de las actitudes, no resulta ser nuevo, aunque plenamente novedoso en el ambiente local, desde la perspectiva de la institución educativa formadora de docentes y en el contexto del carácter académico desarrollado.

\section{La actitud}

Una de las definiciones más representativas de las actitudes es la de Rokeach (1968), mencionado por Leyva Valls y Córdoba Amate (2010), quien las concibe como una organización relativamente duradera de creencias en torno a un objeto o situación, que predispone a reaccionar preferentemente de una manera determinada. (Leyva y Córdoba, 2010)

Según este autor, una actitud es una orientación o disposición aprendida hacia un objeto o situación, que proporciona una tendencia a responder de manera favorable o desfavorable hacia el objeto o situación. El aprendizaje puede no estar basado en la experiencia personal, sino que puede adquirirse a través de aprendizaje observacional e identificación.

Algunos autores, como Berkowitz (1972), proponen dividir en categorías las definiciones con base en tres características básicas. Existen varios grupos los cuales han desarrollado ciertas definiciones para lo que es actitud, algunas de estas definiciones son:

Actitud es una evaluación o una reacción efectiva.

Característica principal de una actitud la disposición de actuar de cierto modo.

Es una mezcla de tres componentes: el afectivo, el cognoscitivo y el conductual. 
Por otra parte, Campbell (1963) propone clasificar las distintas definiciones de actitud en dos categorías:

- Conductistas (disposiciones de respuesta a cualquier disposición que sirva de base a una ejecución aprendida).

- Cuasi fenomenológicas (considera tres componentes fundamentales: afectivos, conductuales y cognoscitivos).

Las actitudes han sido consideradas ya sea como preparación mental o como predisposiciones implícitas que ejercen una influencia general y continua en muchas respuestas evaluativas. Las actitudes son, por lo tanto, internas, eventos privados cuya existencia inferimos de nuestra propia introspección o de alguna forma de evidencia conductual, cuando se expresan abiertamente en palabras o en obras. Una actitud verbalizada se llama opinión.

\subsection{Componentes de la actitud}

De acuerdo con Secord y Backman (1964), citado por Gross (1992), la mayoría de las definiciones de actitud comprenden tres componentes:

El cognoscitivo, lo que una persona cree acerca del objeto de la actitud, incluye el dominio de hechos, opiniones, creencias, pensamientos, valores, conocimientos y expectativas, especialmente de carácter evaluativo, acerca del objeto de la actitud. Destaca en ellos, el valor que representa para el individuo el objeto o situación.

El afectivo, lo que una persona siente acerca del objeto de la actitud, incluye procesos que avalan o contradicen las bases de nuestras creencias, expresados en sentimientos evaluativos y preferencias, estados de ánimo y las emociones que se evidencian, física y/o emocionalmente, ante el objeto de la actitud (tenso, ansioso, feliz, preocupado, dedicado, apenado, etc.)

El conductual, como responde una persona ante el objeto de la actitud con base al cognitivo y al afectivo. Este componente de gran importancia en el estudio de las actitudes que muestran las evidencias de actuación a favor o en contra del objeto o situación de la actitud, amén de la ambigüedad de la relación conducta-actitud.

De igual forma Myers en 1993, explica que las diferentes maneras en que actuamos se relacionan con una actitud, cuando se evalúa dicha actitud se aprovecha una de las tres dimensiones: afecto (sentimientos), conducta (intención) y cognición (pensamientos). La parte afectiva puede ser medida a través de las respuestas del sistema nervioso simpático, 
expresiones verbales de gusto y disgusto. La parte cognitiva por medio de las respuestas preceptúales, expresiones verbales de la creencia, autoevaluación de creencias o por la cantidad de conocimientos que una persona tiene acerca de un tema. El componente conductual se puede medir con observación directa del comportamiento en situaciones específicas de estimulación.

El elemento cognitivo el docente puede manifestar la excelencia académica, el rigor científico y la pertinencia social, el compromiso con sus identidades, roles, tareas, responsabilidades y libertades docentes para el ejercicio profesoral. (Picón, 1986)

El profesor puede seleccionar y variar situaciones de aprendizaje respetando los intereses del estudiante. Conjuntamente, el profesor tiende a ser amplio y flexible cognitivamente para discutir aspectos relacionados con la formación académica, evitando la imposición de criterios deterministas sobre el pensamiento y los requerimientos estudiantiles.

La actitud pedagógica puede evidenciar una competencia docente, facilitando el aprendizaje autónomo en el educando. El profesor tiende a crear, recrear, evaluar y adecuar las estrategias pedagógicas de intervención educativa en forma efectiva y constante; asimismo, puede demostrar una capacidad institucional para articular el contexto general (sociedad) con el contexto específico (universidad); y así, promover el uso de materiales, equipos, actividades, etc.; disponibles en la universidad para realizar la formación profesional. El docente busca la producción cognitiva para abrirse y disponerse a los rápidos cambios dados en el mundo del conocimiento científico, tecnológico, institucional, específicamente, docente; conduciéndolo a estimular y orientar el aprendizaje mediante la formación profesional alternativa. (Bar, 1999)

El elemento afectivo, según Bar (1999), desde lo pedagógico puede ser la capacidad e interés emocional para comunicarse de manera abierta, sobre lo académico y profesional, con profesores y estudiantes. El profesor tiende a tolerar académica y profesionalmente diversos enfoques, teorías y metodologías pedagógicas requeridas por la formación profesional. Lo afectivo promueve la convivencia socio-profesional en la universidad y dispone positivamente al profesor para compartir escenarios y contenidos programáticos con los empresarios.

Lo afectivo puede activar una interdependencia emocional en el docente, permitiéndole construir una red de emociones en las relaciones humanas necesarias para fortalecer la formación profesional junto con la empresa. La afectividad tiende a promover una 
conducta animadora en el proceso de enseñanza-aprendizaje y en el cambio de paradigmas cognitivo-emocionales de los estudiantes para vivenciar la formación académica.

El elemento conductual comprende la disposición para aplicar y dominar diferentes técnicas de participación docente y profesional en la formación profesional, entre ellas: talleres, cursos, seminarios, prácticas profesionales, pasantías, etc., en la formación universitaria alternativa. El profesor tiende a dominar y producir métodos y materiales académicos requeridos constantemente en la formación profesional alternativa, comprobando su efectividad y pertinencia académica. Esto puede permitir al docente producir persistentemente nuevos modelos y contenidos formativos, interactuando con empresarios en la formación profesional alternativa. (Rodríguez, 2000)

El elemento comportamental alude a un dinamismo, sin llegar a la desorientación pedagógica que demostraría el profesor en la formación profesional; además, apunta en el docente a un movimiento laboral y profesional que permita el desplazamiento físico e institucional hacia empresas para la formación profesional, intercambiando experiencias académicas con profesores, alumnos y empresarios.

Además, el docente puede interactuar socio-profesionalmente respetando los mecanismos, procesos y situaciones universitarias; así mismo, considerar la dinámica de la relación académica y de producción (bienes-servicios) entre el sector universitario y empresarial.

\section{La Actitud Pedagógica}

En el ambiente académico, la actitud se desarrolla desde la perspectiva orientativa del docente. En efecto, el educando asimila la configuración enmarcada desde la visión profesional asumida por el docente.

En este aspecto cabe mencionar a Luís Meléndez-Ferrer y Lilian Canquiz, Liliana (2003) que cita Porlán (1995) quien planteó que la actitud pedagógica del profesor se enmarca en un contexto profesional constructivista. Esto suscita una conducta mediadora del cambio conceptual ante los estudiantes; es decir, que, habiendo conocido sus ideas o preconcepciones, el docente puede plantear interrogantes o situaciones imposibles de resolver y, desde ellas, pueda incitarlos a buscar y construir otro concepto, que permita al estudiante obtener un significado más complejo. Igualmente, esta actitud es un proceso psicológico que tiende a promover la internalización y la efectividad de la dinámica 
enseñanza-aprendizaje; y también, conducir al profesor a demostrar una conducta cónsona con un modelo profesional de la docencia, que facilite el aprendizaje mediante la práctica indeterminista, creando círculos virtuosos de aprendizaje. De allí que el modelo docente simulará y proporcionará las herramientas necesarias para el aprendizaje significativo en el estudiante universitario.

Estos autores hacen hincapié en la actitud pedagógica señalando varias concepciones, tomados de diferentes autores: (Meléndez-Ferrer y Canquiz, 2003)

La actitud pedagógica es un proceso reflexivo donde el profesor analiza su práctica docente, la manera de corregir errores, aceptar nuevas concepciones del mundo educativo, profesional y laboral; y, a su vez, de cómo abrirse a las corrientes del pensamiento científico, tecnológico, profesional, etc.

La actitud puede ser una coherencia pragmática y reflexiva; es decir, el docente tiende a reflexionar en y sobre la acción educativa que realiza en su actividad profesional, para ser un investigador dentro del aula.

La actitud pedagógica puede conducir al profesor a seleccionar el conocimiento, métodos y recursos utilizados para cumplir sus funciones educativas, en forma independiente.

El docente tiende a una actitud pedagógica favorable para trabajar en el proceso de enseñanza-aprendizaje con estudiantes y empresarios. Esto conduce al profesor a cambiar sus esquemas de pensamiento sobre el proceso de profesionalización, científicotecnológico, organizacional, y de relaciones interprofesionales, etc.

La actitud es la capacidad del profesor para conocer la teoría que fundamenta su acción pedagógica; y que su conducta profesoral no sólo es transmitir un conocimiento ya estructurado, sino tener una actitud que guíe la construcción de su conocimiento en las experiencias académicas y en las que sea responsable de resolver problemas de aprendizaje. El profesor tiende a generar una disposición pedagógica favorable hacia un nuevo modelo docente; esto permite el trabajo interdisciplinario, en equipo y con responsabilidad compartida (Meléndez-Ferrer y Canquiz, 2003).

\section{ESTRATEGIAS METODOLÓGICAS O MATERIALES Y MÉTODOS}

El trabajo se llevó adelante en el Centro Regional de Educación de Pilar (CREP) Mariscal Francisco Solano López de la ciudad de Pilar, departamento de Neembucú, específicamente en el nivel de formación docente, a fin de conocer la actitud que manifiestan los estudiantes con respecto a la profesión que desarrollarán en el futuro. 
En este contexto, se toma como población a los alumnos de Formación Docente matriculados en el año 2019, que, según los datos proporcionados por las autoridades de la institución, totalizan 50. Debido a este número de alumnos, se considera como muestra para la aplicación de los instrumentos de recolección de datos a la totalidad de la población, es decir 50 alumnos.

Como técnicas de recolección de datos se recurre, a entrevistas y cuestionarios, a más de fuentes secundarias.

Entrevista semi estructurada: Destinada a los estudiantes de formación docente del CREP y a directivos y docentes.

Cuestionario tipo escala o test de Actitud de elaboración propia: A fin de definir el desarrollo de actitudes hacia la profesión por parte de los estudiantes de formación docente del CREP.

El cuestionario, resultante del pretest (el cuestionario que se valida) incluye los ítems que cubren los aspectos de las actitudes. Intenta diferenciar y discriminar entre estudiantes que muestran una actitud más favorable hacia la docencia. La Escala Likert comprende varias proporciones declarativas que expresan un punto de vista sobre los indicadores propios de factores de la actitud en el ámbito pedagógico. Se pide a las personas que indiquen el grado en que concuerdan o no concuerdan con la opinión expresada, distribuyendo los ítems del cuestionario formalmente en las tres dimensiones de la actitud, componente cognitivo 26 reactivos, aspecto conductual 17 reactivos, tendientes a la reproducción y uso de nuevos conocimientos a partir de los contenidos programáticos; y por último el componente afectivo con 8 reactivos, que considera al factor emocional como valor preponderante en la aprehensión de nuevos conocimientos. El uso de la Escala de Likert, responde a los criterios de los ítems establecidos en los tres aspectos de la dimensión actitudinal de la siguiente manera:

\begin{tabular}{|l|c|l|l|}
\hline \multicolumn{3}{|c|}{ Parámetros de los niveles de actitud } \\
\hline 1 & TDA & Totalmente de acuerdo & Altamente favorable \\
\hline 2 & DA & De acuerdo & Favorable \\
\hline 3 & I & Indeciso & Indiferente \\
\hline 4 & ED & En desacuerdo & Desfavorable \\
\hline 5 & TED & Totalmente en desacuerdo & Altamente desfavorable \\
\hline
\end{tabular}

Fuente: Elaboración propia. 


\section{RESULTADOS Y DISCUSIÓN}

Se concluye el trabajo, de conformidad a los resultados alcanzados, teniendo en cuenta los objetivos de las dimensiones de la actitud, el problema de investigación. Finalmente se realiza sugerencias sobre la base de las falencias detectadas de conformidad a los hallazgos de la investigación.

Respecto a las características sociodemográficas de los estudiantes de Formación Docente del Centro Regional de Educación de Pilar sobre la profesión, se concluye que los alumnos del 1er año de Formación Docente son menores de 20 años y más. En su mayoría corresponde al sexo femenino más que el sexo masculino, proceden del casco urbano y un ínfimo porcentaje proceden de zonas rurales. Se dedican a sus estudios y otros además de estudiar se dedican a la enseñanza particular.

En cuanto a la dimensión cognitiva de los estudiantes de Formación Docente del Centro Regional de Educación de Pilar sobre la profesión, a partir de los 19 de 26 reactivos seleccionados del Cuestionario tipo Escala administrado a los mismos; de los Parámetros de los niveles de actitud, se Identifica que, de los 50 alumnos de la muestra, en mayor proporción clasifican la información adquirida, describiendo la información obtenida en el conocimiento poniéndola en sus propia palabras, además que el $45 \%$ de los mismos, optan por discutir la información, explicando la información obtenida en el conocimiento, para así expresar e indicar la información del contenido programático; el 57\% de los alumnos manifiestan ubicar la información; el $62 \%$ reconoce y selecciona la información; $43 \%$ reporta la información; el 57\% Ilustra el conocimiento; el 52\% de los alumnos indican la importancia de interpretar el conocimiento; el $38 \%$ el de analizar el conocimiento; el 50\% contrasta y formula el conocimiento; el 55\% Diagrama el conocimiento y el $45 \%$ de los alumnos indican proponer los conocimientos de los contenidos programáticos; el 55\% argumenta los conocimientos defendiendo su postura respecto a los conocimientos previos.

De esta manera, se hace referencia a un buen desarrollo del reconocimiento de los tres tipos de conocimientos en los estudiantes de la muestra: el que tiene, el que posee y el que puede conseguir, que cuanto más detallado sea el conocimiento más favorable será la actitud positiva hacia la profesión, se refleja resultados favorables.

Con relación a la dimensión conductual de los estudiantes de Formación Docente del Centro Regional de Educación de Pilar sobre la profesión, a partir de los 11 de 17 
reactivos seleccionados del Cuestionario tipo Escala administrado a los mismos; de los Parámetros de los niveles de actitud, se Reconoce que, de los 50 alumnos de la muestra, en mayor proporción el $40 \%$ reproduce los aspectos del conocimiento, el 50\% usa los conocimientos para la formación de actitudes; el 60\% indica preparar el conocimiento para la formación de actitudes; el 50\% manifiestan practicar el conocimiento y 50\% programa el conocimiento para la formación de actitud; el 35\% experimenta el conocimiento en el proceso de actitud pedagógica y el $40 \%$ construye los conocimientos de los contenidos programáticos; el 55\% de los estudiantes manifiestan crear los conocimientos de los contenidos programáticos, el $45 \%$ planea y califican los conocimientos previos en el proceso de actitud pedagógico, y el 40\% apoya el conocimiento para la formación de actitudes. Se concluye así, que se logró reconocer, que de los estudiantes de la muestra comprenden la acción caracterizada por el dominio de la información actualizada y pertinente, el cual permite asimilar contenidos programáticos aplicables en la formación profesional y establecer una comunicación fluida, constructiva y favorable para la interacción formadora entre estudiantes, profesores y ejecución a partir de la pauta institucional.

Respecto a la dimensión afectiva de los estudiantes de Formación Docente del Centro Regional de Educación de Pilar sobre la profesión, a partir de los 6 de 8 reactivos seleccionados del Cuestionario tipo Escala administrado a los mismos; de los Parámetros de los niveles de actitud, se Identifica que, el $45 \%$ indica la importancia del conocimiento previo en el proceso dentro de la actitud; el 50\% relaciona los conocimientos para la formación de actitudes; $53 \%$ escoge el conocimiento para la formación de actitudes; el $47 \%$ valora el conocimiento en el proceso de la actitud pedagógica; $63 \%$ critica el conocimiento en el proceso de dar actitud pedagógica ante la formación profesional; el $53 \%$ en cuanto a la importancia de evaluar el conocimiento previo en el proceso de actitud pedagógica ante la formación profesional.

De lo expuesto en los resultados, se identifica que ésta es la dimensión que requiere mayor atención, ya que en más de un reactivo del cuestionario se ha indicado como indiferente o estar en desacuerdo, en tanto que, se reconoce que el aspecto afectivo es un valor preponderante, que incluye sensaciones y sentimientos expresados hacia la profesión, tiene que ver con la comunicación abierta entre los estudiantes y para con los docentes, 
promoviendo la convivencia social y profesional, activando una interdependencia emocional en las relaciones humanas para fortalecer la formación profesional.

Se Determina que la importancia que los estudiantes de Formación Docente del Centro Regional de Educación de Pilar le dan a la profesión, en su mayoría, es que asume su compromiso social y ético, que implica la profesión, porque también existen algunos que están influenciados por características negativas del contexto globalizado, que no asumen su verdadera importancia.

\section{CONCLUSIÓN O CONSIDERACIONES FINALES}

Con relación a las competencias que demuestran los estudiantes al egresar del Nivel de Formación Docente, se evidencia en primer lugar la actitud investigativa para reflexionar y dar alternativas de solución a los problemas de su entorno social, así también el manejo de lenguas oficiales del país y por último la capacidad para planificar y evaluar los procesos del PEA (Proceso de Enseñanza- Aprendizaje)

Los egresados demuestran dominio de contenidos actualizados y acordes a los nuevos paradigmas pedagógicos, desarrollan habilidades y estrategias innovadoras en procedimientos del PEA y el uso de recursos tecnológicos.

Con relación a la coherencia de las competencias adquiridas y las actividades laborales, cabe destacar que como institución formal, la malla curricular desarrollada responde a un trabajo científico y a la realidad educativa.

En cuanto a la actitud que demuestran los alumnos con respecto a la carrera y a su futura profesión, se destaca que existen estudiantes que demuestran actitudes de responsabilidad, compromiso y espíritu crítico, aunque no en todos los alumnos esto pueda visualizarse. Así también, se destaca la responsabilidad, autogestión, liderazgo, predisposición hacia el trabajo en equipo como actitudes resaltantes en su formación, sin omitir que existe un margen o porcentaje de alumnos que necesitan afianzar ciertos valores o definir sus actitudes positivas, pero que eso no se logra solo en el Nivel de Formación Docente sino durante su carrera profesional.

Otro aspecto que se menciona, es que el Nivel de Formación Docente, como institución formadora, busca el logro del Perfil de salida, o las competencias que deben llevar desarrollados como facilitadores de la enseñanza y proceso de educación. Por lo que los resultados obtenidos de los docentes de la mencionada institución lo ubican en un nivel óptimo, ya que los alumnos adquieren nociones conceptuales y habilidades 
procedimentales, innovadores, a través de profesionales muy destacados en su formación académica y recursos muy actualizados. El nivel de Formación Docente brinda experiencias de práctica profesional, enriquecedoras, que le aproximan a la práctica real de la profesión.

Los resultados de la investigación además permitieron demostrar que:

1. Existe una falla formativa en los alumnos del 1er año de Formación Docente debido a la falta de, primero de Orientación Vocacional al momento de escoger su carrera y posteriormente en lo que a Valores personales y profesionales se refiere.

2. Se observa una clara tendencia hacia el mejoramiento profesional por parte de los alumnos con la finalidad de alcanzar un nivel de especialización que les permita alcanzar los ascensos que les brinda mejoras salariales e incrementar la calidad en la educación.

3. Se hace imperiosa la necesidad de que en las unidades curriculares de los pensum de estudios se incluyan dentro de sus programas la formación en valías

En consideración a lo antes mencionado, es indispensable recalcar que a pesar de estas carencias los alumnos han desarrollado un instinto natural que les ha permitido una cierta capacidad para su desempeño profesional, aunque no la suficiente y esto repercute en la formación de las nuevas generaciones que estará bajo su responsabilidad.

\section{LISTA DE REFERENCIAS}

Bar, G. (1999). Perfil y Competencias del docente en el Contexto

Institucional Educativo. Madrid: Organización de Estados Iberoamericanos para la Educación, la Ciencia y la Cultura.

Instituto Superior de Tecnología de Monterrey (2000). Unidad 8: Las estrategias metodológicas. Disponible en: www.notes.qro.itesm.mx Leyva Valls, M. y Córdoba Amate, C. (2010). Actitudes y valores: cómo trabajarlos en el aula. Rev. Sapiencia. Disponible en http://www.feteugtalmeria.org Meléndez-Ferrer, Luis y Canquiz, Liliana. (2003). La actitud del profesor universitario desde una perspectiva pedagógica, en el marco de la relación universidadempresa. Rev. Ped, set. vol.24, no.71. Disponible en www.scielo.org.ve

Picón, G. (1986). El Proceso de Convertirse en Universidad. Aprendizaje Organizacional en la Universidad Venezolana. Caracas: Fondo Editorial de la Universidad Pedagógica Experimental Libertador (FEDUPEL). 
Rodríguez, R. (2000). El aula de clase convertida en un ambiente de

aprendizaje significativo. Disponible

en:

www.geocities.com:0080/Athens/8478/rosaadelina.htm

Romero Barea, G. (2010). Experiencia educativa: la actitud del profesor en

el aula. Revista digital: innovación y experiencia. Córdoba

Tonucci, F. (1999). La Investigación como Alternativa a la Enseñanza.

Caracas: Laboratorio Educativo

Vega, M. (2000). Contextualización de la intersectorialidad de la relación

entre la academia y la empresa a través de los enfoques teóricos que la explican. OMNIA.

Zúñiga, M. (1994). Información de Educación. Del Constructivismo al

Construccionismo. Disponible en: www.mep.go.cr/educacion/constructivismo.asp

http://www.nfdcrep.org/ 